\title{
The Application of Optimized ICA Method Towards Endmember Extraction
}

\author{
Zuo-wei Huang ${ }^{1,2, *}$, Min-hua Yang ${ }^{1}$ and Yu Zou ${ }^{2}$ \\ ${ }^{1}$ School of Geosciences and Information-Physics, Central South University, Changsha 410083, China; ${ }^{2}$ School of Archi- \\ tecture and Urban planning, Hunan University of Technology, Zhuzhou 412008, China
}

\begin{abstract}
The advent of hyperspectral image technology is a major leap in recent years, it obtains the surface of the earth image contains rich space, radiation and spectral information, Mixed pixels not only effects identification and classification precision of object, but also greatly hinder the development of quantitative remote sensing, so effectively interpret mixed pixels is an important problem for its applications. Based on optimized ICA method a novel hyperspectral unmixing approach is proposed in the paper, which introducing the similarity threshold technique to describe the statistical distribution of the pixels, and determine the criterion of candidate endmembers, A multi-core parallel processing method is proposed to increase its efficiency. The approach is capable of self-adaptation, and can be applied to hyperspectral images with different characteristics. Experimental results on both simulated and real hyperspectral data demonstrated that the proposed approach can provided an effective technique for the blind unmixing and obviously increase the processing efficiency and obtain accurate results at the same time.
\end{abstract}

Keywords: Endmember extraction, hyperspectral image, ICA, parallel processing, spectral Unmixing.

\section{INTRODUCTION}

With the deep development of quantification, there were wide concerns regarding the hyperspectral remote sensing technology both at home and abroad. Thanks to the high spectral resolution and images integrated with the spectral information of the hyperspectral imagery [1-3] it has been gradually deepening from qualitative analysis to quantitative analysis. However, due to limited spatial resolution and features of the complex diversity, on the ground of the regional distribution of residential complex, Spectral unmixing is the key technique in hyperspectral remote sensing analysis. Endmember extraction is the most important and complicated procedure in spectral unmixing $[4,5]$. Spectral unmixing can acquire the appropriate endmembers and abundances according to minimizing the residual sum of Squares, because of the large quality of hyper spectral image bands and strong correlation between adjacent ones. Redundancy is inevitably in the observed data [6-8], therefore, it needs to reduce the dimension of images. Base on the development and research status of ICA, it carried on a systematic study of noise reduction and dimension reduction methods for hyper spectral data. The Linear Spectral Mixture Model (LSMM) is one of the Mixed Decomposition Models (MPD) that has been applied extensively. Based on the hypothesis of the existence of the pure pixels, the Endmember Extraction Algorithms (EEAs) can be categorized into two classes:
Endmember Identification Algorithm(EIA) and Endmember Generation Algorithm(EGA). Recently, many state-of-the-art parallel EEAs have been developed, including the parallel nonnegative matrix factorization (NMF) algorithms (Stefan, 2006; Dong, 2010), parallel endmember extraction algorithms and parallel automated morphological endmember extraction (P-AMEE) (Plaza, 2006) [9-11]. The techniques of blind signal separation (BSS) and projection pursuit (PP) are introduced into EE and enriched statistics based EEAs $[12,13]$. Independent component analysis (ICA) is a classic spectral unmixing BSS algorithm (Bayle's, 1997; Chang, 2002; Nascimento, 2005), which is also used in EE (Wang, 2006). Spatial information incorporating EEAs, aiming at including spatial information in the process of endmember extraction, have been widely developed, such as automated morphological endmember extraction (AMEE) (Plaza, 2002), spatial-spectral endmember extraction (SSEE) (Rogge, 2006) and spatial preprocessing (SPP) method (Zortea \& Plaza, 2009) [14-16]. Solving the problem of the mixed can not only acquire high-precision classification and sub-pixel object reorganization, but also ensure more adequate usage of the hyperspectral image. The image aims to use high spectral band information from the pixel level to enter the sub-pixel level, to decompose the basic unitendmember of the mixed pixel, and to calculate the proportion of these endmembers. By making pretreatment spectral unmixing, object classification and recognition are more accurate, so there is a broad space for object classification, mineral prediction, urban layout analysis, chemical composition and detection $[17,18]$. Spectrums from different endmembers are assumed as independent and mixed pixels are linearly mixed by independent components. Therefore the ICA can separate abundance maps for different endmembers. 


\section{ENDMEMBER EXTRACTION ALGORITHM}

Mixed pixel decomposition (MPD) which is the most effective method for solving the mixed pixel problem can break through the limitation of spatial resolution, obtain the property information of the mixed pixels on a sub pixel precision level and improve the classification precision. As we all known LSMM is one of the mixed decomposition model that been applied most extensively, it mainly includes four parts (Fig. 1): Data Preprocessing, Endmember Extraction, Abundance Estimation and Accuracy Assessment, its expression can be presented as below:

$x=\sum_{i=1}^{p} A_{i} S_{i}+\varepsilon=A S+\varepsilon$

where $p$ is the number of the endmembers, $A$ is the endmember matrix of $n \times m$, its every column is endmember vector of image, called endmember spectral set, $\mathrm{S}$ is the component ratio of endmember composition for every pixel, $A_{i}$ and $\mathrm{S}_{\mathrm{i}}$ is the $\mathrm{i}$-th endmember and mixing proportion, respectively, and $\varepsilon$ represents the random noise term. In terms of the physical significance, Eq.(1) expresses that in hyperspectral image, any pixel vector can be expressed as a combination of $\mathrm{n}$-dimension component ratio by $\mathrm{n}$-dimension endmember vector, its mathematical meaning is a product of matrix $\mathrm{P}$ consisting of $\mathrm{m} \times \mathrm{n}$ dimension endmember vector and vector $\mathrm{s}$ consisting of $\mathrm{m}$-dimension component ratio.

There are two constraints for the abundance vector: nonnegativity constraints (ANC, Abundance Non negativity Constraint, $\mathrm{s}_{\mathrm{i}} \geq 0, \mathrm{i}=1,2, \ldots, \mathrm{p}$ ) and as the Constraint (ASC, Abundance Sum to one Constraint,

$$
\left.\sum_{i=1}^{p} s_{i}=1, i=1,2, \ldots, p\right)
$$

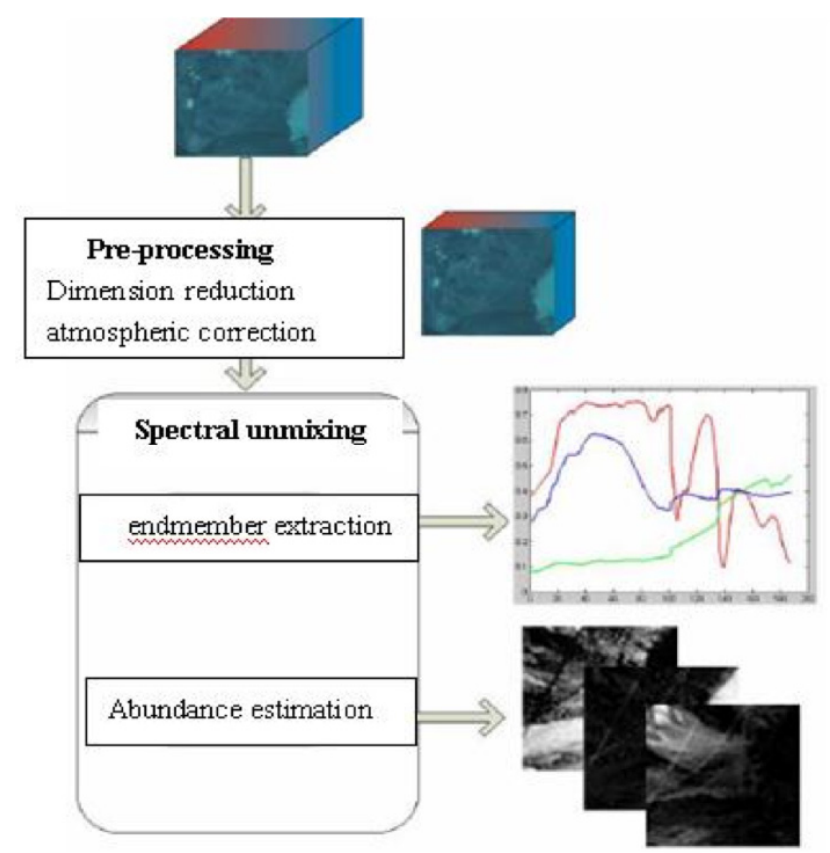

Fig. (1). The processing of Mixed pixel decomposition.

It shows that confirming matrix $\mathrm{A}$ is the key, not only from the view of the physical significance but also the mathematical significance. The spectral vectors forming ma- trix A must have a physical significance for the image, namely it must be the most probable endmember. Then, matrix A must have stability in mathematics, namely not illconditioned matrix.

A maximum volume simplex endmember extraction algorithm based on the volume calculation method of the simplex in the original spectral space is proposed. In view of the relationship of the LSMM and the convex simplex theory, it uses the volume calculation method of the convex simplex that does not need the dimension reduction procedure implemented into the N-FINDR and SGA algorithms, based on convex geometry method, the N-FINDR algorithm looks for pure a pixel set in the imagery, the algorithm considers: in L dimension spectrum Space, which is composed by pure pixel of monomer volume which is larger than any other pixel $[4,5]$. The algorithm starts from a set of random pixel set, and ongoing monomer "inflation" in data internal, for each pixel, each endmember, endmember will be replaced by pixel spectrum, and its volume was recounted. If the volume increases, endmember will be replace by pixel spectrum, this has not happen until now and no alternative has been repeated so far. The algorithm is shown below:

(1) Preprocessing: Using the dimension reduction methods such as PCA transform to reduce the dimensionality to be $\mathrm{p}-1$.

(2) Exhaustion search: for any monomer which is composed of pixel spectral vector (number p), select the pixels randomly as the initial endmembers, and calculate the simplex volume $V_{1}$ that is composed of these initial endmembers. The mathematical dentition of the volume is as follows:

$$
V\left(A_{1}, A_{2}, \ldots, A_{p}\right)=\frac{1}{(p-1) !} \mid \operatorname{det}\left[\begin{array}{cccc}
1 & 1 & \ldots & 1 \\
A_{1}^{\prime} & A_{2}^{\prime} & \ldots & A_{p}^{\prime}
\end{array}\right]
$$

where $A_{i}(i=1,2, \ldots p)$ where $A_{i}$ is the $p-1$ dimensional column vector for the $\mathrm{i}$-th endmember looking for the monomer volume's biggest vector sets $\left\{A_{1}^{*}, A_{2}^{*}, \ldots, A_{p}^{*}\right\}$, its expression can be presented below as:

$$
\left\{A_{1}^{*}, A_{2}^{*}, \ldots, A_{p}^{*}\right\}=\arg \left\{\begin{array}{c}
\max \\
\left\{A_{1}, A_{2}, \ldots, A_{p}\right\}
\end{array} \quad V\left(A_{1}, A_{2}, \ldots, A_{p}\right)\right\}
$$

(3) Evaluate an image pixel by replacing one pixel in the represented endmember set with the image pixel $\mathrm{P}_{1}$, producing a"trial enemember"set and calculate the new simplex volume $V_{2}$. If $V_{2}>V_{1}$, then replace the candidate enemember with $\mathrm{P}_{1}$

(4) Replace the other candidate enemembers with the pixel P, execute the procedures (2) and (3) for all the other pixels repeatedly. The simplex volume is composed of result where endmembers is the largest one, and its vertexes correspond to the endmembers.

\section{OPTIMIZED ICA MODEL FOR UNMIXING HY- PERSPECTRAL IMAGE}

\subsection{ICA Model}

ICA is a signal processing method originated from blind source recently and finds underlying components which are 


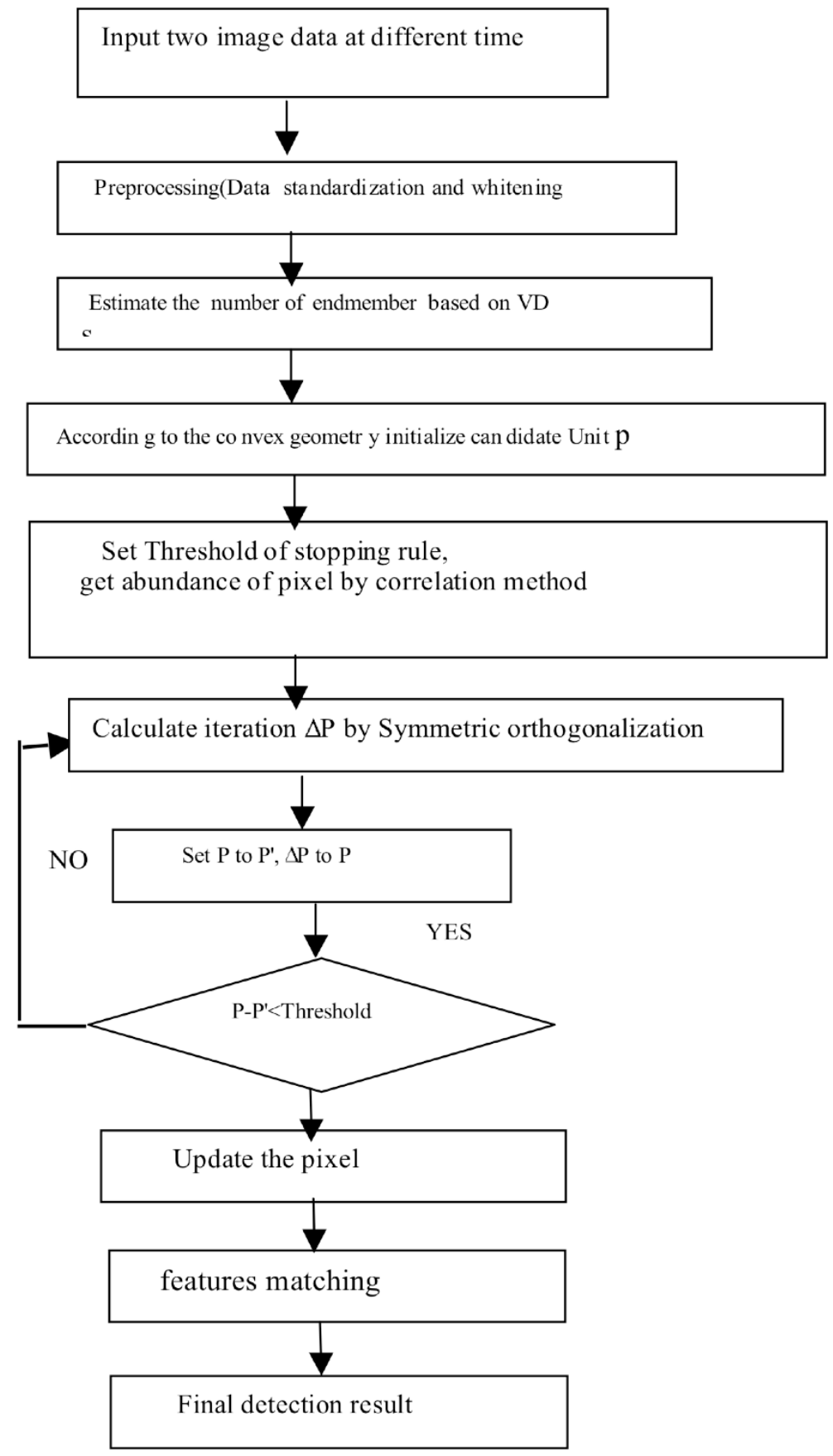

Fig. (2). Flow chart of spectral information detection.

both independent and non-gaussian from multidimensional statistical data. Through a linear transformation, it enables independence for each other's source of signals. Independent components are separated by finding the projection direction with maximum Gaussian property, it assumes that the composite signal is mixed by several signals which are both statistically independent and non-gaussian. Using several observations, ICA can separate independent.

Signals. Assume that $\mathrm{n}$ observations $\mathrm{y}_{\mathrm{i}}(\mathrm{t})$ are composited of unknown source signal $\mathrm{x}_{\mathrm{j}}(\mathrm{t})$. Observations can be presented as below.

$y_{i}(t)=\sum_{j} w_{i j} x_{j}(t), i=1, \ldots, n ; j=1, \ldots, m$ where $w_{i j}$ is the abundance of $j$ source signal in $i$ observation. ICA is a process that is used to find $\mathrm{w}_{\mathrm{ij}}$ for unknown source signal $x_{j}(t)$. There are two uncertainties in the model: one is not being sure regarding the order of the independent components, the other is not deciding the variances of the independent component. Spectral information detection flow chart is shown in Fig. (2).

Based on non-gaussianity Characteristics ICA finds a separation matrix $\mathrm{W}$, aiming to find the maximum limit of the non-gaussianity measured by the high order statistics and the approximation of negentropy, which makes the convergence is fast and reliable. It is referred to the previous work of Hyvärinen (1999) which is represented by: 


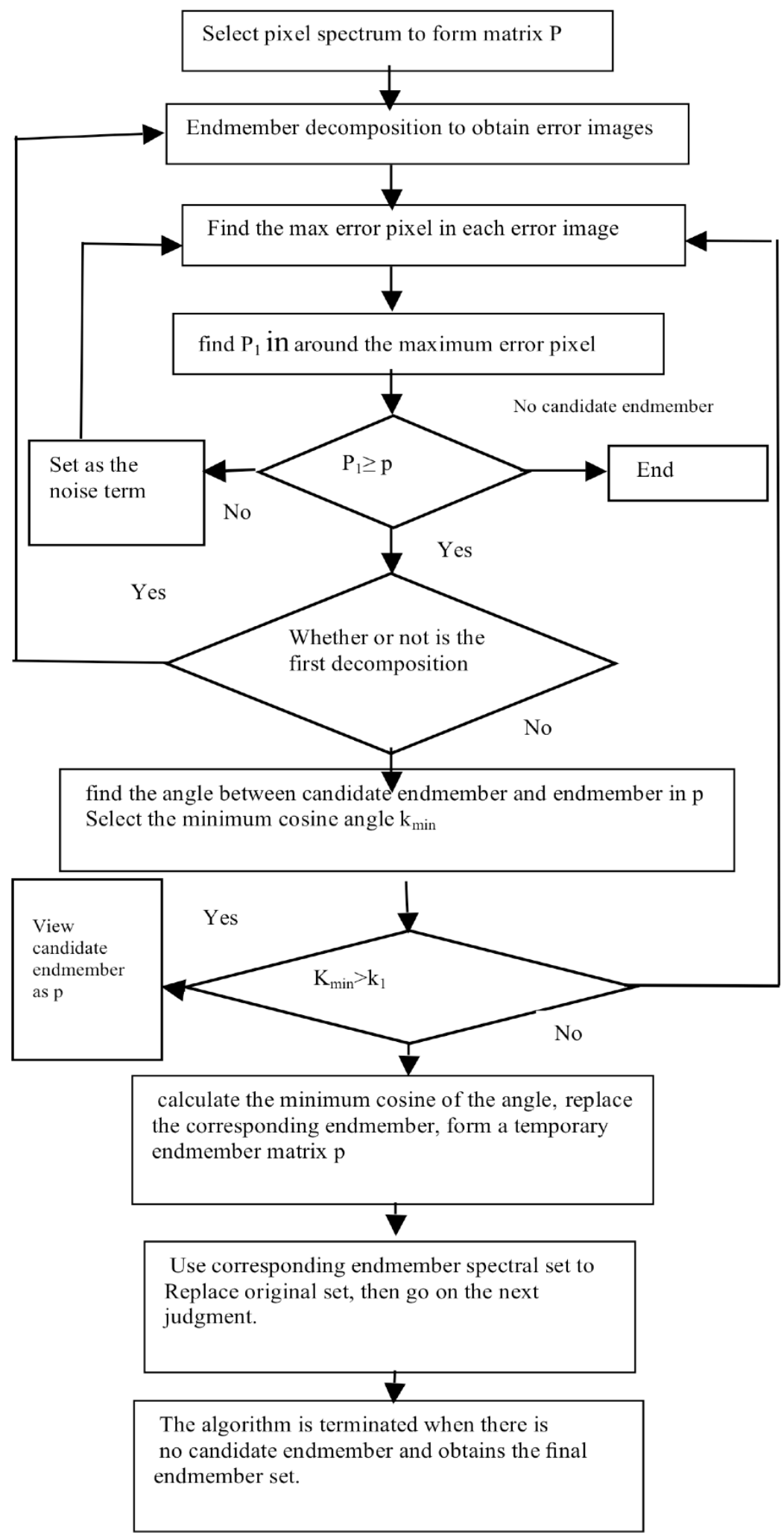

Fig. (3). The judgment rule chart of candidate endmember.

$W^{(k+1)}:=E\left\{g\left(W^{(k) T} Y\right)\right\}-E\left\{g^{\prime}\left(W^{(k) T} Y\right)\right\} W^{(k)}$

where $\mathrm{W}^{(\mathrm{k}+1)}$ represents the new matrix of $\mathrm{W}^{(\mathrm{k})}$ in the $(\mathrm{k}+1)$ the iteration ICA has been widely used in digital image feature extraction, speech processing, biomedical signal processing, and other field, etc.

\subsection{The Criterion of Candidate Endmember}

Owing to the large quality of hyperspectral image bands, strong correlation between adjacent ones, redundancy is inevitably for data, it needs to remove the noise and reduce the dimension, as a result the spectral and spatial de-correlation 
method was put forward. The number of abundance in ICA process equals to the number of independent components resulting from separating the endmember change. However, as a result of large number of noise and several endmembers being contained in hyperspectral difference images, we should calculate more abundance than the number of endmembers in order to avoid losing change of information in reprocessing. Firstly it divides the error image into many spatial regions according to the continuity of image the features, secondly the residual standard deviation is obtained by spectral dimension of the multiple linear regressions, select the pixel vector with maximum error in every region. And lastly the judgment rule for candidate's endmember is used to select candidate endmember.

Since spectrum and noise in spectral similarity has special relation, it is assumed that the spectral angle function of hyperspectral imagery is $\mathrm{V}(\mathrm{x}, \mathrm{y})$, as a result the variogram of the spectral angle can be described as

$$
y(h)=\frac{1}{2 N} \sum_{i=1}^{N}\left[\left(V(x, y)-V\left(x^{\prime}-y^{\prime}\right)\right]^{2}\right.
$$

where $\mathrm{h}$ is the Euclidean distance between pixel $(\mathrm{x}, \mathrm{y})$ and pixel $\left(x^{\prime}, y^{\prime}\right)$. $N$ refers to the amount of two pixels which are separated by $h$. calculating the spectral angle takes central pixel's spectrum in window as the reference spectrum, in order to improve the sensitivity for distinguishing the endmember spectrum and noise pixel, assume the threshold of pixel's similarity is $\mathrm{K}_{1}$ and the threshold for pixel's nonsimilarity is $K_{2}$. Because endmember in its near range has similarity with the mixed spectrum, but noise has no similarity with its near pixel spectrum, so $K_{1}$ and $K_{2}$ is taken to measure noise and endmember separately. It not only takes full advantage of mixed spectrums near characters but also gets rid of the noise's interference on selecting endmember. The flow chart of proposed algorithm is shown in Fig. (3).

\subsection{Optimized ICA Algorithm}

As we all known the algorithm of hyperspectral unmixing has high computational complexity, In order to enhance the efficiency of the processing, it needs to apply parallel processing of hyperspectral image, and the symmetrical multi-Processing (SMP) cluster which is deemed as one of the most popular parallel architecture with the development of multi-core CPU era and computer network. Taking advantage of the shared memory and distribution memory of the parallel model, the efficiency can be improved by attaching threads, called thread elements (TEs), the processes executed by each SMP nodes provide a scalable inter-node parallelism. These executive inter-node processes are called process elements (PEs) which can achieve better scalability and providehigher computing performance for endmember extraction.

According to above theory, optimized ICA EEA is given in the following steps:

Step 1: Select two hyperspectral images at different time, according to the rule of non-noise pixels with Maximum difference, apply two levels of data partitioning strategy into the image to get difference in image and matrix $\mathrm{W}$, which is initialized in step 2;
Step 2: The master PE randomizes a group of numbers with uniformed distribution, based on external correlation feature of $\mathrm{W}$, which it collects via candidate's endmembers to prevent the ill-condition of matrix and increase its stability;

Step 3: Pixels data is standardized (meaning its value equals to zero), as a result the Whitening matrix is defined by:

$V=D^{-\frac{1}{2}} E^{T}$

where $\mathrm{D}=\operatorname{Diag}\left(\mathrm{d}_{1}, \ldots, \mathrm{d}_{\mathrm{n}}\right)$ is diagonal matrix with eigen values of covariance of difference image and $\mathrm{E}=\left(\mathrm{e}_{1}, \ldots, \mathrm{e}_{\mathrm{n}}\right)$ is composed by eigenvectors of covariance of different image as columns. In this way the master PE image initialize and obtain $\mathrm{W}^{(\mathrm{k})}$;

Step 4: The master PE de-correlate and renormalizes $\mathrm{W}^{(\mathrm{k}+1)}$ after each iteration, and judges whether the endmember matrix is the first to undergo decomposition, if so, then it goes on with the unconditional decomposition. Otherwise it starts judgment on the next value.

Step 5: The master collects all $\mathrm{W}^{(\mathrm{k})}$, initializes its iteration value with unit-norm columns and then perform the symmetric orthogonalization. It is done in order to calculate all the projection vectors at the same time on the basis of the theory that differentiates the independent components which are orthogonal in whitening space. The advantage of symmetric orthogonalization is that the calculation error will not be accumulated as a result of parallel calculation. Symmetric orthogonalization is stated as follows: by:

$$
\begin{aligned}
& w \leftarrow w\left(w^{T} w\right)^{-\frac{1}{2}} \\
& w\left(w^{T} w\right)^{-\frac{1}{2}}=E \cdot \operatorname{diag}\left(d_{1}^{-\frac{1}{2}}, \ldots, d_{m}^{-\frac{1}{2}}\right) E^{T}
\end{aligned}
$$

where $\mathrm{W}$ represents projection matrix, $\operatorname{diag}\left(d_{1}^{-\frac{1}{2}}, \ldots, d_{m}^{-\frac{1}{2}}\right)$ is eigenvalue matrix of $\left(\mathrm{W}^{\mathrm{T}} \mathrm{W}\right)$, and $\mathrm{E}$ is the matrix composed by corresponding eigenvectors.

Step 6: Based on spectral similarities measure weight gives an appropriate weight for each pixel, if $\mathrm{K}_{1}<\mathrm{K}_{2}$, then that means that the candidate's endmember and endmember in $\mathrm{W}$ has slight external correlation and enough differences. The judging process regarding whether the candidate endmember can enter the next Iteration, is given as follows:

$\Delta P=\frac{X\left(\left(P^{t} X\right)^{2}\right)^{T}}{N}$

where $\mathrm{P}\left(\mathrm{p}_{1}, \ldots, \mathrm{p}_{\mathrm{m}}\right)$ is $\mathrm{K} \times \mathrm{M}$ projection matrix, $\mathrm{X}=\left(\mathrm{x}_{1}, \ldots, \mathrm{x}_{\mathrm{N}}\right)$ is $\mathrm{K} \times \mathrm{N}$ image matrix, $\mathrm{N}$ is pixel number and square means square of every element in matrix.

Step 7: A judgment is made to ensure the stability of the EEA, it tries to seek for the candidate endmember in the physical meaning It's solved in the iterative calculation process of algorithm, and makes the matrix $\mathrm{P}$ closer to complete and stable status after the optimized iteration calculation, it broadcasts to all PEs until it satisfy the convergence condition. 
Step 8: Every PEs in pixels group decompose their data partitioning it uniformly, after which they set the convergence error, Calculate difference value of matrixes before and after iteration. If the difference value is greater than the error, iteration starts again from Step 4, otherwise the final iteration result is obtained.

Step 9: Using data partitioning method all PEs execute Step 5and Step 7, image is projected with projection matrix $\mathrm{W}$ which is obtained by iteration with maximum Gaussian to get abundance of independent components.

Step 10: The master PE is executed to obtain the final endmember set.

\section{EXPERIMENTS AND ANALYSIS}

\subsection{The General Condition of the Study Area and Data Collection}

The study area is located in the central part of Hunan Province in china, covering Changsha city and the surrounding areas $\left(1026 \mathrm{~km}^{2}\right)$, the scene size is of $200 \times 150$ pixels, the real hyperspectral data collected by the AVIRIS was acquired on August 23,2012 and August 25,2012, it covers the spectral region from $0.4-2.5 \mu \mathrm{m}$ in 224 bands with a $10 \mathrm{~nm}$ band width and a $20 \mathrm{~m}$ spatial resolution. To improve the unmixing performance, we removed the low SNR bands, the abundance of the pixel in the data was created randomly, and in the meantime, the abundances of each pixel fulfill the ANC and ASC was replaced by the pixels whose maximum abundance was larger than 0.8 with a mixture made up of all endmembers of equal abundances. To simulate possible errors and sensor noise, it added zero-mean Gaussian noise to the mixture data. The image data was converted into the reflectance data using the ATREM method, the remnant error was minimized using the EFFORT method. The false color image of the study area is illustrated in Fig. (4).

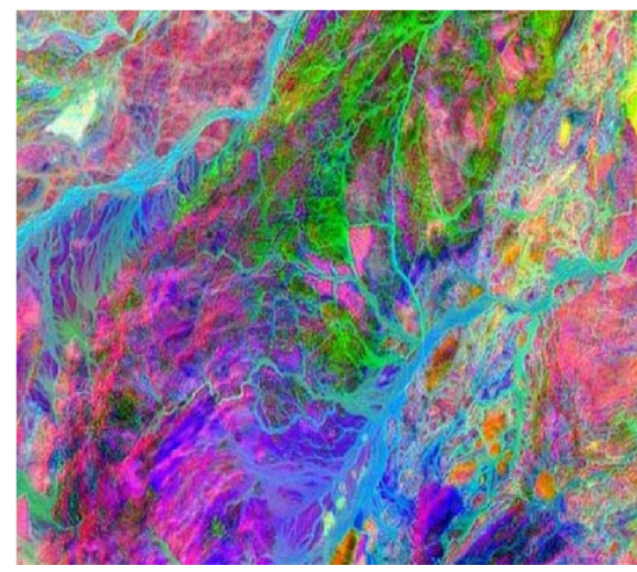

Fig. (4). Image of the study area.

The system runs on Windows XP operating system, based on MPICH2 message passing library the testing platform consists of 25 nodes connected by Ethernet. Each node has $2 \mathrm{GHz}$ Intel Core Duo CPU, and uses Visual C++ 2008 OpenMP supported compiler for SMP cluster environment.

In order to quantitatively evaluate the algorithm this paper generates simulated hyperspectral data, contrasting the simulated.
Data for the classic endmember extraction algorithm $(\mathrm{N}-$ FINDR and ICA) and the optimized algorithm (O-ICA) in the experiments and tests them in the same conditions, aiming to evaluate the extraction results, it adopts the following three statistical indicators: root mean square error (RMSE), Kappa Coefficient and false alarm rate. The indicators of quantitative study use Spectral angle distance (SAD), spectral information divergence (SID), Abundance Information Divergence (AID) and Bivariate Distribution Function(BDF) to assess the performance of the endmember extraction.

\subsection{Endmember Extraction Experiment and Analysis}

In this experiment, four objects including plant, Water body, road, and building were taken from USGS spectral library and were used as endmembers to construct mixed pixels. According to above Optimized ICA algorithm endmember spectral curves are given in Fig. (5), seen from the spectrum, four end members are not identical each other.

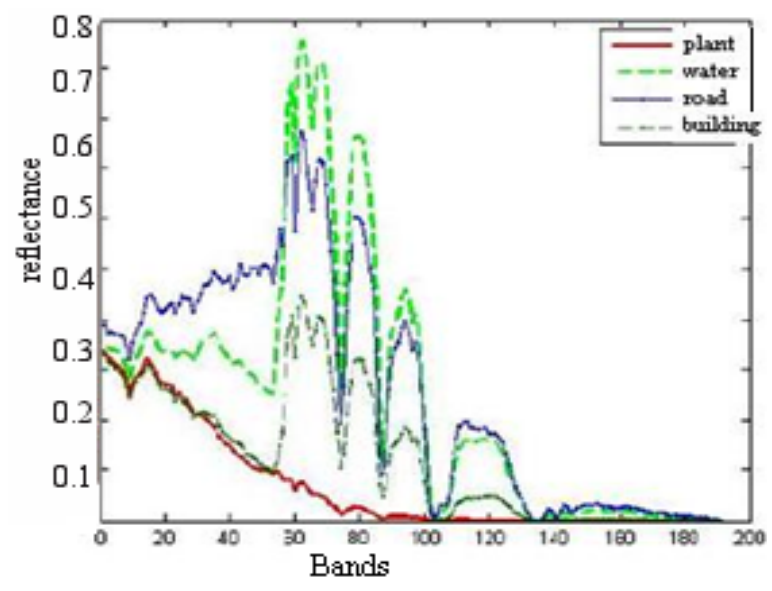

Fig. (5). Endmember extraction result.

Apart from the endmember extraction results from the visual system, the paper also uses the comparison method from reference spectra, which is selected from a piece of pure land (pure pixel), the comparison chart is shown in Fig. (6), The angle cosine value between endmember extraction.

Result and reference for the spectral are shown in Table 1, it can be seen that the endmember extraction accuracy is rather good from spectral angle cosine value.

Bivariate Distribution Function (BDF) is a kind of visual evaluation method for mixed pixels.

It used $\mathrm{X}$-axis represents the true abundance, $\mathrm{Y}$-axis represents the estimated abundance. For good estimate result, the scatter points should be located in a straight line of $y=x$, since the influence of the estimation error and some other factors, the reasonable estimate Points should be0 located around in the line of $y=x$.

It can be seen from the Fig. (7), these four objects points all fall in the range less than $8 \%$ and the RMSE of abundance is rather small, so the decomposition accuracy of mixed pixels is higher.

The original N-FINDR method and ICA method were used to extract the four endmembers. SAD and SID was given in Table 2 to assess the accuracy of the extracted 


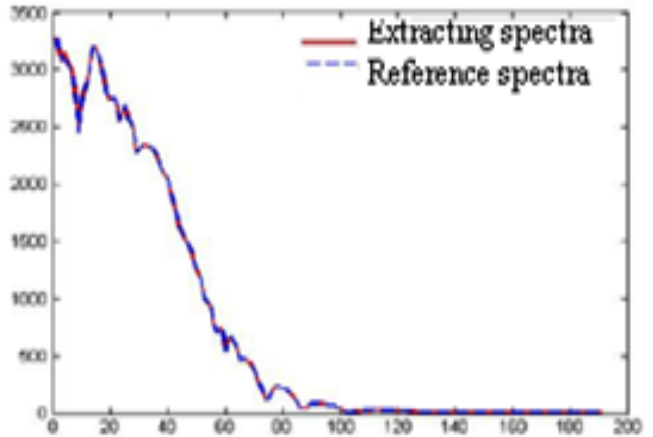

plant

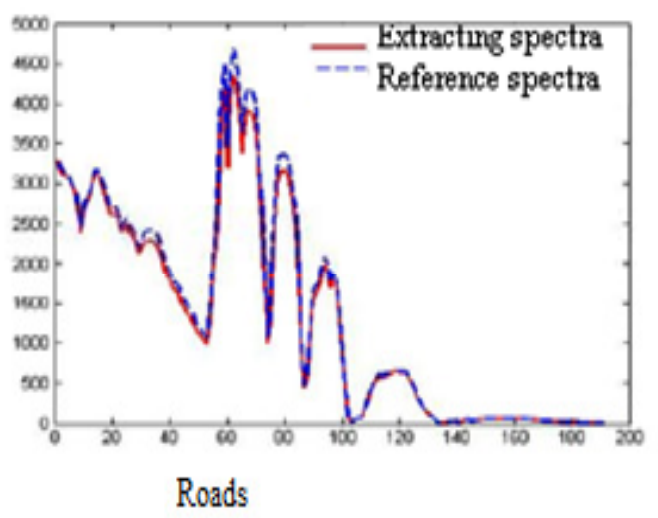

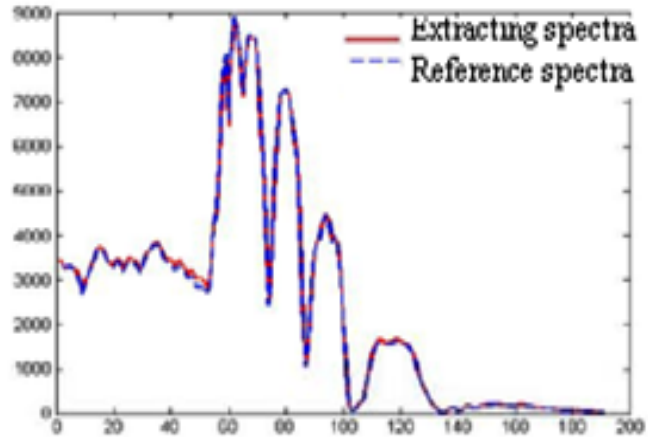

water body

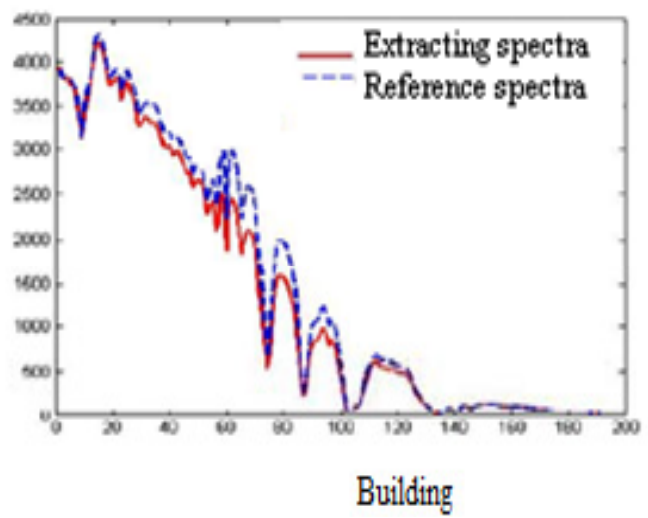

Fig. (6). Compare results of extraction endmember and reference spectra.
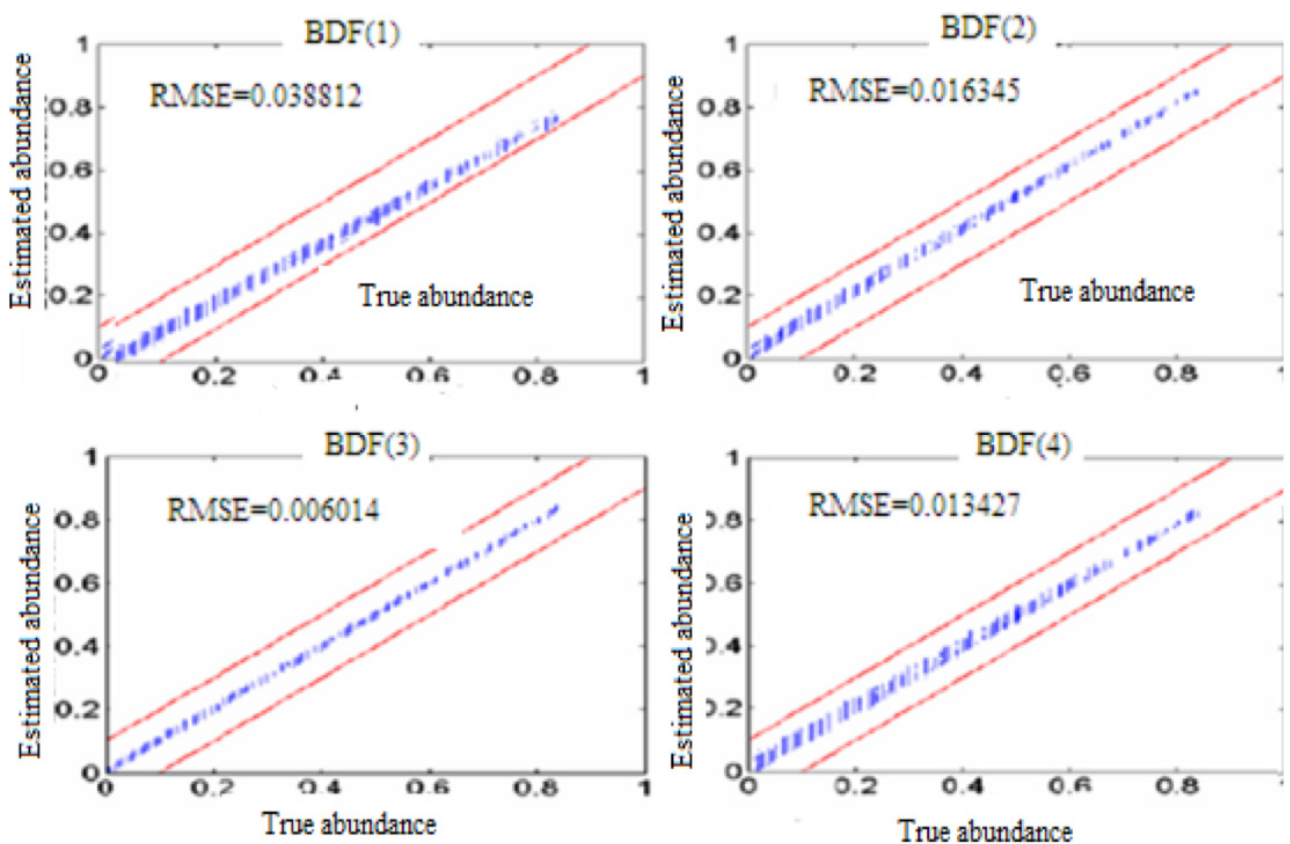

Fig. (7). The distribution of Bivariate Distribution Function.

Table 1. The comparison of Endmember and reference spectra.

\begin{tabular}{|c|c|c|c|c|}
\hline Endmember & $\mathbf{E}_{\mathbf{1}}$ & $\mathbf{E}_{\mathbf{2}}$ & $\mathbf{E}_{\mathbf{3}}$ & $\mathbf{E}_{\mathbf{4}}$ \\
\hline \hline cosine value & 0.9923 & 0.9998 & 0.9978 & 0.9995 \\
\hline
\end{tabular}


Table 2. Accuracy assessment of the extracted endmembers.

\begin{tabular}{|c|c|c|c|c|}
\hline & $\mathbf{E}_{\mathbf{1}}(\mathbf{S A D} / \mathbf{S I D})$ & $\mathbf{E}_{\mathbf{2}}(\mathbf{S A D} / \mathbf{S I D})$ & $\mathbf{E}_{3}(\mathbf{S A D} / \mathbf{S I D})$ & $\mathbf{E}_{\mathbf{4}}(\mathbf{S A D} / \mathbf{S I D})$ \\
\hline \hline N-FINDER & $0.0923 / 0.0331$ & $0.1365 / 0.0536$ & $0.0872 / 0.0069$ & $0.2315 / 0.0478$ \\
\hline ICA & $0.0902 / 0.0326$ & $0.1372 / 0.0498$ & $0.0802 / 0.0063$ & $0.2324 / 0.0472$ \\
\hline O-ICA & $0.0872 / 0.0301$ & $0.1206 / 0.0431$ & $0.0779 / 0.0060$ & $0.2072 / 0.0405$ \\
\hline
\end{tabular}

Table 3. Classification accuracy (SVM).

\begin{tabular}{|c|c|c|c|c|}
\hline Objects Indicator & Buildings & Water & Roads & Plant \\
\hline \hline Kappa Coefficient & $79.66 \%$ & $83.84 \%$ & $88.67 \%$ & $89.69 \%$ \\
\hline Recognition Precision & $88.24 \%$ & $83.26 \%$ & $83.97 \%$ & $87.48 \%$ \\
\hline false alarm rate & $4.28 \%$ & $4.57 \%$ & $3.88 \%$ & $2.16 \%$ \\
\hline
\end{tabular}

Table.4. Classification accuracy (O-ICA).

\begin{tabular}{|c|c|c|c|c|}
\hline Objects Indicator & Buildings & Water & Roads & Plant \\
\hline \hline Kappa Coefficient & $90.78 \%$ & $89.23 \%$ & $92.53 \%$ & $95.69 \%$ \\
\hline Recognition Precision & $92.56 \%$ & $85.86 \%$ & $87.37 \%$ & $90.13 \%$ \\
\hline false alarm rate & $1.26 \%$ & $2.27 \%$ & $0.98 \%$ & $1.11 \%$ \\
\hline
\end{tabular}

endmembers. It showed that o-ICA algorithms had better accuracy than other two extracted methods.

Aim to verify the objects classification accuracy based on O-ICA method, using the confusion matrix method, take Kappa coefficient and Recognition Precision and false alarm rate as an index, in order to enhance the contrast, SVM is also employed to classify the same region. The classification results are listed in Table $\mathbf{3}$, at the same time the classification results after unmixing (O-ICA) are listed in Table 4, which indicate that both methods can provide satisfactory classification results. The total accuracy of the classification is $93.22 \%$, it show that $4.8 \%$ higher than that of SVM. The Kappa and Recognition Precision of this method is also higher than that of ML method.

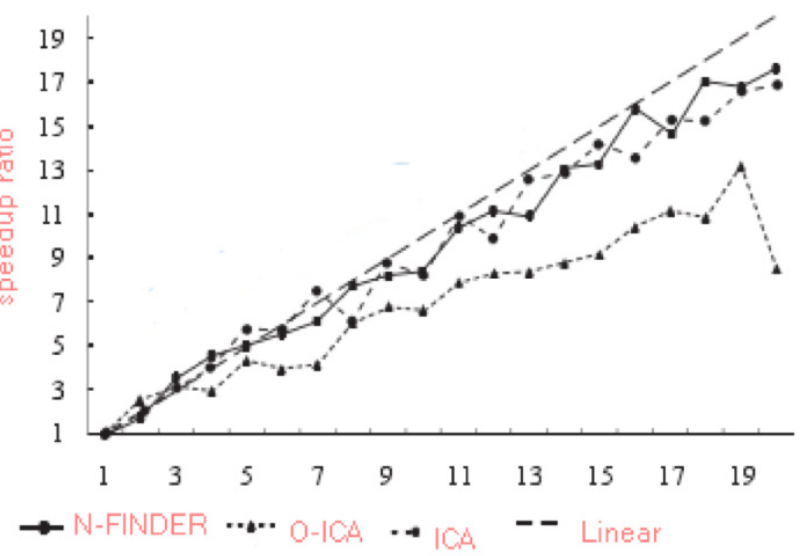

Fig. (8). The speedup ratio curves.
The speedup radio curves for our proposed algorithms are shown in Fig. (8). The result indicates that the speedup radio of O-ICA is higher than ICA and N-FINDER method. From the consuming time, we can see that the efficiency order of the EEAs from high to low is: O-ICA, N-FINDR and ICA.

\section{CONCLUSION}

High-resolution images usually have high spatial resolution and can provide more information of the cover types. The mixed pixels bring a big problem in interpretation and hinder the development of remote sensing technology. It has been found that the endmember extraction accuracy is affected by several factors namely: segmentation scale, endmember number and abundance constraint degree. Coming up with the conception of digital earth, hyperspectral remote sensing technology plays an increasingly important role in modern science and technology, which can be used to obtain a two-dimensional object distribution information and onedimensional spectral feature characteristic information at the same time. According to the hyperspectral data, while extracting the pure pixel respects single features, it could also classify these pixels. Therefore, the extracting endmembers is one of the keys to hyperspectral remote sensing technology. The data feature of hyperspectral data having several hundreds of bands results in huge amounts of computing. So the question arises, how the analysis of data and the extraction of endmembers became one of the key problems in hyperspectral remote sensing image processing? The answer is that Under the condition of the same extracted bands, O-ICA 
can reserve more information of the original images to improve the accuracy of target classification of remote sensing images, and the advantage of the algorithm has been further verified. ICA is based on the assumption of mutually independent sources, which violates the constraint conditions in LSMM. This compromises of ICA applicability to hyperspectral data. To overcome this problem, it introduces a solution for the minimization of total correlation of the components. Interestingly, with the minimization of total correlation of the components, the angle of the direction between each component is invariable. The performance of the proposed algorithm was verified using both the synthetic hyperspectral data experiments and the real hyperspectral data experiments. It was found that with the increase of overall number of pixels, the computation time increased quickly, while the computation time of the newly proposed O-ICA methods was much less than that of original algorithm. It also demonstrates high parallel performance and greatly improves the performance of O-ICA endmember extraction. The set of pixels that maximizes the volume of the simplex are selected as endmembers, because the initial set is chosen randomly, the obtained endmember set may be different after multiple sequential runs of algorithm. The performance of the proposed algorithm was verified using both the synthetic hyperspectral data experiments and the real data experiments. It was found that with the increase of overall number of pixels, the computation time of it is increased quickly, while the computation time of the newly proposed method was much less than that of original algorithm.

\section{CONFLICT OF INTEREST}

The authors confirm that this article content has no conflict of interest.

\section{ACKNOWLEDGEMENTS}

The authors would like to thank the anonymous reviewers for their careful reading of this paper and for their helpful comments, This work is supported by the National Natural Science Foundation of China (No. 61273157) and Geographic Spatial Information Engineering Key Laboratory of the State Bureau of Surveying and mapping (No. 201215).

\section{REFERENCES}

[1] C. I. Chang, C. C. Wu, W. M. Liu and Y. C. Ouyang, "A new growing method for simplex-based endmember extraction algorithm",. IEEE Transactions on Geosciences and Remote Sensing, vol. 44, no. 10 , pp. 2804-2819, 2012.
[2] J. C. Qi, S. L. Zhu, B. S. Zhu, Y. Zhao and E. S. Li, "Research on automated end member extraction algorithm", Hydrographic Surveying and Charting, vol. 29, vol. 2, pp.16 -19, 2009.

[3] L. D. Miao and H. E. Qi, "Endmember extraction from highly mixed data using minimum volume constrained nonnegative matrix factorization", IEEE Transaction on Geoscience and Remote Sensing, vol. 45, no. 3, pp. 765-777, 2007.

[4] J. M. P. Nascimento and J. M. Dias. "Vertex component analysis: A fast algorithm to unmix hyperspectral data", IEEE Transactions on Geoscience and Remote Sensing. vol. 43, no. 4, pp. 34-38, 2005.

[5] D. C. Heinz. "Real-time processing of an Unsupervised constrained linear spectral unmixing algorithm" In //IGARSS01. Geoscience and Remote Sensing Symposium, 2011, 372 -374.

[6] J. Wang and C. I. Chang. "Independent component analysis-based dimensionality reduction with applications in hyper spectral image analysis", IEEE Transactions on Geoscience and Remote Sensing, vol. 44, no. 6, pp.1586-1600, 2006.

[7] H. T. Du, H. R. Qi and X. L. Wang. "A parallel independent component analysis algorithm". In Proceedings of the 12th International Conference on Parallel and Distributed Systems. Minnesota: IEEE Computer Society Press, 2006, pp.151-160.

[8] L. P. Zhang and X. Huang. "Advanced processing techniques for remotely sensed imagery", Journal of Remote Sensing, vol. 13, no. 4, pp. 559-569, 2012.

[9] J. Q. Zhong and R. S. Wang. "Multi-temporal remote sensing change detection based on independent component analysis". International Journal of Remote Sensing, vol. 27, no. 10, pp. 2055$2061,2013$.

[10] A. Plaza, J. A. Benediktsson, J. W. Boardman, J. Brazile, L. Bruzzone, J. C. Tilton and G. Triani, "Recent advances in techniques for hyperspectral image processing", Remote Sensing of Environment, vol. 113, no. 1, pp. S110-S122, 2009.

[11] M. Zortea and A. Plaza, "Spatial preprocessing for endmember extraction", IEEE Transactions on Geoscienceand Remote Sensing, vol. 47, no. 8, pp.2679-2693, 2009.

[12] J. Wang and C-I. Chang. "Applications of independent component analysis in end member extraction and abundance quantification for hyperspectral imagery", IEEE Transactions on Geoscience and Remote Sensing, vol. 44, no. 9, pp. 2601-2616, 2006.

[13] L. M. Zhang, S. C. Chen, L. H. Qiao. "Graph optimization for dimensionality reduction with sparsity constraints". Pattern Recognition, vol. 45, no. 3, pp.1205-1210, 2012.

[14] D. Cai, X. F. He and J. W. Han, "Spectral regression for dimensionality reduction". Technical Report UIUCDCS-R-2007-2856, Computer Science Department, UIUC, May 2007.

[15] S. J. Li and H. R. Qi, "Sparse representation based band selection for hyperspectral datas". In Proceeding of 18th IEEE International Conference on Image Processing, Brussels, 2011, pp.11-14.

[16] M. Hasanlou, F. Samadzadegan. "Comparative study of intrinsic dimensionality estimation and dimension reduction techniques on hyperspectral datas using K-NN classifier", IEEE Geoscience and Remote Sensing Letters, vol. 9, no. 6, pp.1046-1050, 2012.

[17] G. Hughes, "On the mean accuracy of statistical pattern recognizers", IEEE Transactions on Information Theory, vol. IT-14, no. 1, pp. 55-63, 1968.

[18] C. I. Chang and H. Safavi, "Progressive dimensionality reduction by transform for hyperspectral datary", Pattern Recognition, vol. 44, no. 10/11, pp. 2760-2773, 2011.

Received: September 22, 2014

Revised: November 30, 2014

Accepted: December 02, 2014

(C) Huang et al.; Licensee Bentham Open.

This is an open access article licensed under the terms of the Creative Commons Attribution Non-Commercial License (http://creativecommons.org/licenses/by-nc/3.0/) which permits unrestricted, non-commercial use, distribution and reproduction in any medium, provided the work is properly cited. 